\title{
Participation of the endoplasmic reticulum protein chaperone thio-oxidoreductase in gonadotropin-releasing hormone receptor expression at the plasma membrane
}

\author{
W. Lucca-Junior, J.A. Janovick and P.M. Conn \\ Division of Neuroscience and Reproductive Sciences, Oregon National Primate Research Center, \\ Oregon Health and Science University, ONPRC/OHSU, Beaverton, Oregon, USA \\ Correspondence to: W. Lucca-Junior, Rua Pedro Pacheco, 3, Cohab II, 14750-000 Pitangueiras, SP, \\ Brasil \\ Fax: +55-16-3633-0017. E-mail: wlucca1@hotmail.com
}

\begin{abstract}
Chaperone members of the protein disulfide isomerase family can catalyze the thiol-disulfide exchange reaction with pairs of cysteines. There are 14 protein disulfide isomerase family members, but the ability to catalyze a thiol disulfide exchange reaction has not been demonstrated for all of them. Human endoplasmic reticulum protein chaperone thio-oxidoreductase (ERp18) shows partial oxidative activity as a protein disulfide isomerase. The aim of the present study was to evaluate the participation of ERp18 in gonadotropin-releasing hormone receptor (GnRHR) expression at the plasma membrane. Cos-7 cells were cultured, plated, and transfected with $25 \mathrm{ng}$ (unless indicated) wild-type human GnRHR (hGnRHR) or mutant GnRHR (Cys ${ }^{14} \mathrm{Ala}$ and $\mathrm{Cys}^{200} \mathrm{Ala}$ ) and pcDNA3.1 without insert (empty vector) or ERp18 cDNA (75 ng/well), pre-loaded for $18 \mathrm{~h}$ with $1 \mu \mathrm{Ci}$ myo-[2$3 \mathrm{H}(\mathrm{N})$ ]-inositol in $0.25 \mathrm{~mL}$ DMEM and treated for $2 \mathrm{~h}$ with buserelin. We observed a decrease in maximal inositol phosphate (IP) production in response to buserelin in the cells co-transfected with hGnRHR, and a decrease from 20 to $75 \mathrm{ng}$ of ERp18 compared with cells co-transfected with hGnRHR and empty vector. The decrease in maximal IP was proportional to the amount of ERp18 DNA over the range examined. Mutants (Cys ${ }^{14}$ Ala and Cys ${ }^{200} \mathrm{Ala}$ ) that could not form the Cys ${ }^{14}$-Cys ${ }^{200}$ bridge essential for plasma membrane routing of the hGnRHR did not modify maximal IP production when they were co-transfected with ERp18. These results suggest that ERp18 has a reduction role on disulfide bonds in wild-type hGnRHR folding.
\end{abstract}

Key words: Protein chaperones; Gonadotropin-releasing hormone receptor; Calnexin; Calreticulin; ERp18

Research supported by NIH grants HD-19899, RR-00163, HD-18185, and TW/HD-00668.

Received February 18, 2008. Accepted December 11, 2008

After synthesis in the endoplasmic reticulum (ER), G protein-coupled receptors (GPCRs) are often folded and assembled to be packaged into the ER-derived COPIIcoated vesicles and transported through the Golgi apparatus and the trans-Golgi network in order to arrive at the plasma membrane. During transportation through the ER and Golgi structures, GPCRs are submitted to post-translational modifications to acquire the mature conformation (1). These events - folding and trafficking of newly synthe- sized proteins - are highly regulated processes that likely require a number of different chaperone molecules belonging to the cell's quality control system (QCS). These QCS chaperones recognize non-native conformations of newly synthesized proteins and prevent their aggregation and export of the incompletely folded chains from the ER (2). When the maturation of a newly synthesized protein is aborted or inefficiently performed, chaperones catalyze a covalent bond between ubiquitin and the unfolded protein. 
This reaction targets misfolded proteins to proteosomal degradation by the ER-associated degradation process (3-5). A large number of diseases are associated with degradation of misfolded proteins such as Parkinson's, Alzheimer's, hypogonadotropic hypogonadism, diabetes insipidus, and others (6). When the human gonadotropinreleasing hormone receptor ( $h \mathrm{GnRHR}$ ), the smallest representatives of this GPCR superfamily of receptors, is not expressed in the cell's plasma membrane because it was retained in the ER or eventually degraded in the cytosol, its normal function (activation of luteinizing hormone release) is not performed and this results in the disease hypogonadotropic hypogonadism (1,7). Hypogonadotropic hypogonadism is characterized by 1 ) complete or partial absence of any endogenous $\mathrm{GnRH}$-evoked luteinizing hormone pulsations, 2) delayed sexual development, and 3) normalization of pituitary and gonadal function in response to physiological regiments of exogenous $\mathrm{GnRH}$ replacement (8).

Chaperones are an interesting potential therapeutic target because of their role in the cellular QCS, regulating the folding and assembly of newly synthesized proteins, including hGnRHR (1). They are present in the ER, mitochondria and cytoplasm, and comprise a wide class of proteins that may be categorized into five groups: the heat shock protein family, lectins, substrate-specific proteins, protein disulfide isomerases (PDI) and peptidyl prolyl isomerases (6). Each group of chaperones has a different

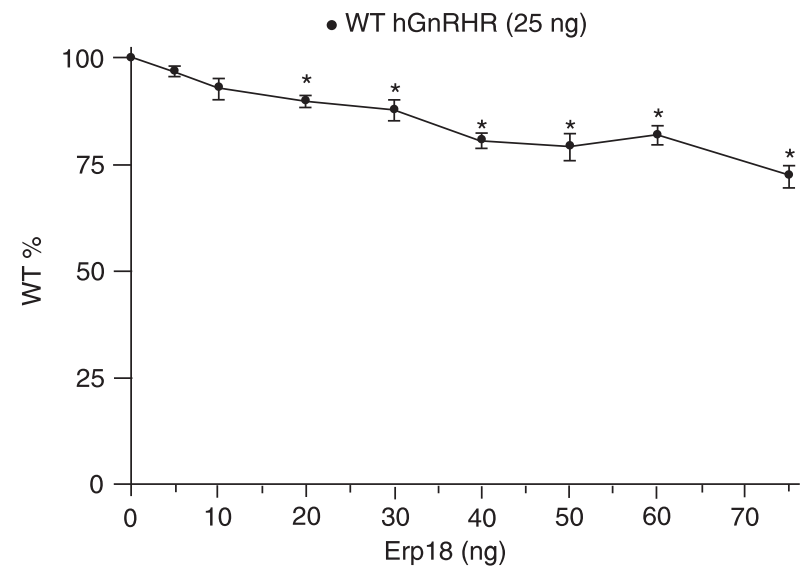

Figure 1. Wild type percent (WT\%) indicates the inositol phosphate production upon buserelin stimulation of WT human gonadotropin-releasing hormone receptor ( $h \mathrm{GnRHR}$ ) co-transfected with the ERp18 chaperone. Data are reported as mean \pm SEM of at least three independent experiments in triplicate incubations. ${ }^{*} \mathrm{P}<0.05$ compared with WT hGnRHR (25 ng) without ERp18 co-transfected (one-way ANOVA followed by the Bonferroni post-test). chemical means to retain, refold and assemble misfolded proteins or promote their eventual degradation. One of these groups is the PDI family of chaperones, which recognize and catalyze the formation and isomerization of nonnative disulfide bonds (9).

PDI family members can catalyze the thiol-disulfide exchange reaction with a pair of cysteines that are frequently arranged in a Cys- $X-X-C y s$ motif (where $X$ is any amino acid). There are 14 PDI family members, but the ability to catalyze a thiol disulfide exchange reaction has not been shown for all members. Some members like PDIr, ERp72, P5, PDIp, ERp28, and ERp18 have the same or partial oxidative activity as PDI, while only ERp57 has the ability to reduce disulfide bonds $(10,11)$.

The aim of the present study was to evaluate the participation of the human endoplasmic reticulum protein chaperone thio-oxidoreductase (ERp18) in gonadotropinreleasing hormone receptor expression at the plasma membrane.

Cos-7 cells were cultured, plated, and transfected, as previously reported (12), with $25 \mathrm{ng}$ (unless indicated) WT hGnRHR, or mutant GnRHR (C14A and C200A) and pcDNA3.1 without insert (empty vector) or ERp18 cDNA (75 ng/well), as indicated, and $1 \mu \mathrm{L}$ lipofectamine in 0.125 $\mathrm{mL}$ OPTI-MEM (room temperature), according to manufacturer instructions. Empty vector (pcDNA3.1, without insert) was included to bring the total cDNA to a final concentration of $100 \mathrm{ng} /$ well, which provides optimal transfection efficiency $(12,13)$. Five hours after transfection, $0.125 \mathrm{~mL}$ DMEM containing 20\% fetal bovine serum and $20 \mu \mathrm{g} / \mathrm{mL}$ gentamicin were added to the wells. Twentythree hours after transfection, cells were washed, then preloaded for $18 \mathrm{~h}$ with $1 \mu \mathrm{Ci}$ myo-[2-3H(N)]-inositol in 0.25 $\mathrm{mL}$ DMEM (prepared without inositol (12)). The cells were then washed twice with $0.25 \mathrm{~mL}$ DMEM containing $5 \mathrm{mM}$ $\mathrm{LiCl}$ (without inositol), treated for $2 \mathrm{~h}$ with $0.25 \mathrm{~mL}$ of the indicated buserelin concentration in the same medium ( $\mathrm{LiCl}$ prevents inositol phosphate (IP) degradation). Total IPs were determined as described previously $(12,14)$. Data are averaged from at least three independent experiments (15).

We observed a decrease in maximal IP production in response to buserelin in the cells co-transfected with hGnRHR and from 20 to 75 ng ERp18 compared with cells co-transfected with hGnRHR and empty vector (Figure 1). The decrease in maximal IP was proportional to the amount of ERp18 DNA over the range examined. These results suggest the participation of ERp18 in GnRHR folding and assembly.

Wild-type hGnRHR has two disulfide-bond bridges Cys ${ }^{114}-{ }^{1}$-ys ${ }^{196}$ and Cys ${ }^{14}-$ Cys $^{200}$. All mutants without the 
disulfide bridge (Cys ${ }^{114}-$ Cys $^{196}$ ) remain in the ER and can never be rescued by IN3 (a pharmacological chaperone with the ability to rescue unfolded proteins by correcting their routing) $(7,16,17)$. However, the Cys ${ }^{14}$-Cys ${ }^{200}$ bridge can be rescued by IN3 (17). In our experiments, mutants that could not form the Cys ${ }^{14}$ - Cys ${ }^{200}$ bridge (i.e., Cys ${ }^{14} \mathrm{Ala}$ and Cys ${ }^{200} \mathrm{Ala}$ ) did not change the maximal IP production when they were co-transfected with ERp18 (Figure 2), but when this bridge was present (wild-type hGnRHR), the cotransfection with ERp18 decreased maximal IP production. These results suggest that ERp18 has a reduction effect on the disulfide bond in wild-type hGnRHR folding. Conversely, in vitro studies (18) have demonstrated that the reduced form of ERp18 is more stable than the oxidized form, suggesting that it is involved in disulfide bond formation. Moreover, they also have demonstrated that ERp18 is relatively inefficient in catalyzing oxidoreductase reactions ( $15 \%$ of a domain of PDI) (18), probably because it lacks the glutamic acid proton acceptor (11). However, inside the ER there are many different oxidoreductases with a range of abilities to catalyze oxidoreductase reactions. Then, even with a relatively inefficient power to catalyze an oxidoreductase reaction there are many possibilities of interaction between ERp18 and other oxidoreductase proteins to reduce the disulfide bond in wild-type hGnRHR.

\section{References}

1. Conn PM, Ulloa-Aguirre A, Ito J, Janovick JA. G proteincoupled receptor trafficking in health and disease: lessons learned to prepare for therapeutic mutant rescue in vivo. Pharmacol Rev 2007; 59: 225-250.

2. Ellgaard L, Helenius A. Quality control in the endoplasmic reticulum. Nat Rev Mol Cell Biol 2003; 4: 181-191.

3. Brodsky JL. The protective and destructive roles played by molecular chaperones during ERAD (endoplasmic-reticulum-associated degradation). Biochem J 2007; 404: 353363.

4. Meusser B, Hirsch C, Jarosch E, Sommer T. ERAD: the long road to destruction. Nat Cell Biol 2005; 7: 766-772.

5. Dong C, Filipeanu CM, Duvernay MT, Wu G. Regulation of $G$ protein-coupled receptor export trafficking. Biochim Biophys Acta 2007; 1768: 853-870.

6. Ni M, Lee AS. ER chaperones in mammalian development and human diseases. FEBS Lett 2007; 581: 3641-3651.

7. Janovick JA, Brothers SP, Cornea A, Bush E, Goulet MT, Ashton WT, et al. Refolding of misfolded mutant GPCR: post-translational pharmacoperone action in vitro. Mol Cell Endocrinol 2007; 272: 77-85.

8. Seminara SB, Hayes FJ, Crowley WF Jr. Gonadotropinreleasing hormone deficiency in the human (idiopathic hypogonadotropic hypogonadism and Kallmann's syndrome):

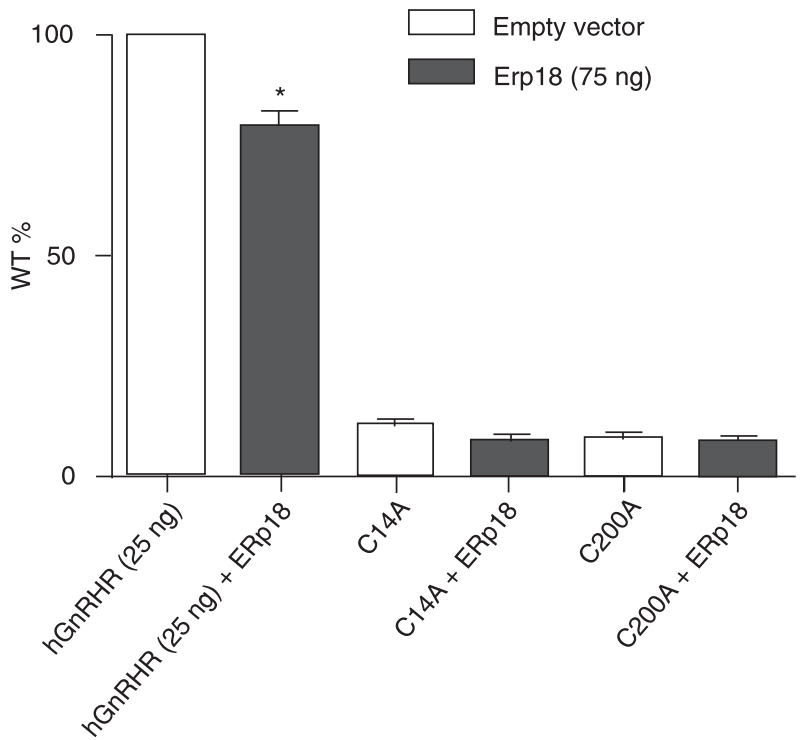

Figure 2. Wild type percent (WT\%) indicates the inositol phosphate production upon buserelin stimulation of WT human gonadotropin-releasing hormone receptor ( $h G n R H R)$ and mutant GnRHRs (C14A and C200A) co-transfected with the ERp18 chaperone. Data are reported as mean \pm SEM of at least three independent experiments in triplicate incubations. ${ }^{*} \mathrm{P}<0.05$ compared with WT hGnRHR (25 ng) without ERp18 co-transfected (one-way ANOVA followed by the Bonferroni post-test).

pathophysiological and genetic considerations. Endocr Rev 1998; 19: 521-539.

9. Jessop CE, Chakravarthi S, Watkins RH, Bulleid NJ. Oxidative protein folding in the mammalian endoplasmic reticulum. Biochem Soc Trans 2004; 32: 655-658.

10. Schwaller M, Wilkinson B, Gilbert HF. Reduction-reoxidation cycles contribute to catalysis of disulfide isomerization by protein-disulfide isomerase. J Biol Chem 2003; 278: 71547159.

11. Ellgaard L, Ruddock LW. The human protein disulphide isomerase family: substrate interactions and functional properties. EMBO Rep 2005; 6: 28-32.

12. Brothers SP, Cornea A, Janovick JA, Conn PM. Human loss-of-function gonadotropin-releasing hormone receptor mutants retain wild-type receptors in the endoplasmic reticulum: molecular basis of the dominant-negative effect. $\mathrm{Mol}$ Endocrinol 2004; 18: 1787-1797.

13. Janovick JA, Ulloa-Aguirre A, Conn PM. Evolved regulation of gonadotropin-releasing hormone receptor cell surface expression. Endocrine 2003; 22: 317-327.

14. Huckle WR, Conn PM. The relationship between gonadotropin-releasing hormone-stimulated luteinizing hormone release and inositol phosphate production: studies with calcium antagonists and protein kinase $\mathrm{C}$ activators. Endocri- 
nology 1987; 120: 160-169.

15. Zhu BT. The competitive and noncompetitive antagonism of receptor-mediated drug actions in the presence of spare receptors. J Pharmacol Toxicol Methods 1993; 29: 85-91.

16. Ulloa-Aguirre A, Janovick JA, Leanos-Miranda A, Conn PM. Misrouted cell surface $\mathrm{GnRH}$ receptors as a disease aetiology for congenital isolated hypogonadotrophic hypogonadism. Hum Reprod Update 2004; 10: 177-192.

17. Janovick JA, Knollman PE, Brothers SP, Ayala-Yanez R,
Aziz AS, Conn PM. Regulation of G protein-coupled receptor trafficking by inefficient plasma membrane expression: molecular basis of an evolved strategy. J Biol Chem 2006; 281: 8417-8425.

18. Alanen HI, Williamson RA, Howard MJ, Lappi AK, Jantti HP, Rautio SM, et al. Functional characterization of ERp18, a new endoplasmic reticulum-located thioredoxin superfamily member. J Biol Chem 2003; 278: 28912-28920. 